\title{
The Surgical Pathology Specimen Safety Study: Management Errors in the Operating Room
}

Semra Bulbuloglu1*, Fatma Eti Aslan' and Meryem Yavuz van Giersbergen ${ }^{2}$

${ }^{1}$ Bahcesehir University, Health Sciences Institute, Istanbul, Turkey

${ }^{2}$ Ege University, Health Sciences Institute, Izmir, Turkey

\begin{abstract}
Background: Surgical Pathology Specimen Management (SPSM) is a collaborative process essential part of patient safety in the operating room. Safe SPSM is important to providing accurate diagnosis and effective treatment for surgical patients.

Aim: The aim of this study is to observe the practice of SPSM in an education and research hospital.

Data sources: Recommended practices of AORN about SPSM was reviewed and a questionnaire form was prepared and conducted. One day survey of surgical pathology material is comprised of 4 excisions, 10 resections a total of 14 specimens in this study. Researcher were observed SPSM process that reported 25 cases.

Results: No process is used for SPSM by the hospital. Identification of patient and specimen information was being in an electronic format. Also, specimens have been held in a shelving unit at room temperature for about 20 to 23 centigrade degrees. Surgical specimen errors were defined in all stages of the SPSM process, in the intraoperative phase during specimen ordering (8\%), labeling $(6 \%)$, containment $(4 \%)$, transport or storage $(40 \%)$, and collection $(8 \%)$. It was determined that the most common contributing parameters were mistakes in communication errors, staff carelessness, information deficit, and lack of policies/protocols issue.
\end{abstract}

Conclusion: The result of this study was reached scientifically evidence about SPSM process was proved insufficient conditions.

Keywords: Material management; Nursing; surgical pathology; Surgical material management

\section{Introduction}

The Association of perioperative Registered Nurses (AORN) previously standardized suggestions on quality control and quality guarantee in the operating room [1-3]. Management of surgically removed specimens is a multifaceted, multidisciplinary process for intraoperative team. Activities in this process consist of accurate site identification, specimen collection, handling, transfer, containment, identification, labeling, preservation, transport, and documentation also perioperative nurses play a crucial role in the process [1]. Specimens are often excised for the diagnosis and treatment of a particular condition and special attention and care should be given to handling surgical specimens [2]. Therefore; correct diagnosis of diseases is a necessary goal for patient safety $[1,2]$. In process of surgical pathology specimen management (SPSM), prevention of possible risk and mistakes is an essential requirement because specimen-related errors reduce the chance of definite diagnosis as well as it was required new interventions and extra procedures $[3,4]$. Besides that, inaccurate SPSM cause increased the risk of mortality, morbidity, high-priced health care [4-6]. In the literature review, it was determined that the most common events recorded during the SPSM with inaccurate patient descriptive information, mislabeling or unlabeled sample, specimen and requisition discordance and unsafe transport [5,7-9]. The interest in safe patient care has greatly increased in recent years [7]. Because surgical specimens are mislabeled, improperly conserved, or lost, the outcomes can be critical, especially when specimens are irremovable [7]. Although it is known significance of effective SPSM, the process cannot be ideally continued both in Turkey and in the World [5]. In a meta-analysis, SPSM errors were determined at the mistake rate of incorrect and incomplete entry of patient information $0.05 \%$ during labeling as well as $7.7 \%$ due to misspelled/in completed/loss of result report in 1.859.402 surgical pathology specimens [6]. However, mislabeling cases are indicated at a ratio of $0.04-0.1 \%$ by the College of American Pathologists reports [8].
Effective SPSM an essential condition in providing patient safety in the operating room. Wrong and deficient applications are reduced the health care quality, also generally the preventable mistakes related SPSM. Consequently, it is very important to identify and prevent possible risks which system, organization and human problems [1-5]. Easy understanding of the spaces SPSM mistakes should be developed the necessary solutions in forestalling the appear of these mistakes. It was determined the types and the contributing factors during the intraoperative phase about SPSM process for give an idea. In this study reviewed cases provide an important information network, a guide for intraoperative team members and raise awareness.

Research Questions: What are the common mistakes in the SPSM process? What causes these?

\section{Methods}

\section{Objective}

In this study; it was aimed to describe the errors of SPSM, and the contributing parameters during the intraoperative phase.

\section{Design}

This point prevalence used a prospective observation of adverse events in the operating room of an education and research hospital in

*Corresponding author: Semra B, Health Sciences Institute, Bahcesehir University, Istanbul, Turkey, Tel: +902123810000; E-mail: semrabulbuloglu@ hotmail.com

Received December 22, 2017; Accepted January 10, 2018; Published January 15,2018

Citation: Bulbuloglu S, Eti Aslan F, van Giersbergen MY(2018) The Surgical Pathology Specimen Safety Study: Management Errors in the Operating Room. Surgery Curr Res 8: 305. doi: 10.4172/2161-1076.1000305

Copyright: (c) 2018 Bulbuloglu S, et al. This is an open-access article distributed under the terms of the Creative Commons Attribution License, which permits unrestricted use, distribution, and reproduction in any medium, provided the original author and source are credited. 
Southeast Anatolian Region in Turkey, on 30th May, 2016.

\section{Setting and sample}

It was operated surgery 77 on patients during one day. During the study, SPSM process was observed by the researchers. It observed of 14 pathology specimens in which consisting 4 excision and 10 resection materials. Therefore, working environment and perioperative team members work style were observed in the operating room. It was followed by using sterile techniques, identification, placement to appropriate solutions and containers, accurate labeling, transportation by highly educated personnel, maintaining patient and employees and accuracy of the records. All of the interventions realized by perioperative team members and support personal were followed without knowing about this study and every initiative was noted by the researcher. Then the records were evaluated by all the researchers. The results of this study were compared to the guidelines published by Association of perioperative Registered Nurses (AORN). Data analysis were handled and interpreted numerically. This study was carried out the hospital that depends on Adiyaman University Presidency Medical Faculty Biomedical Investigations Ethical committee, it was taken on 25th May, 2015 and 2016/4-1 numbered a letter of ethical. It was met with one manager nurse, three intra-operating room nurses who agreed to participate in this study approval in order to review SPSM.

\section{Intervention}

This research is based on observation. No attempt was made.

\section{Results}

It was observed that 4 excisions, 10 resections during this point prevalence survey in the operating room. Number of surgical procedures were 77 patients during survey prevalence of day, at the same day, number of 14 surgical pathology specimens were removed; in these specimens were involved 1 piece mass excision on back, 2 pieces nevus excision, 1 piece pilonidal sinus excision, 3 pieces transurethral resection (TUR-M), 1 piece laparoscopic cholecystectomy, 2 pieces transurethral resection of the prostate (TUR-P), 2 pieces carinal resection-adenoid cystic carcinoma, 1 piece gastric sleeve resection, 1 piece resection soft tissue tumor. The defining 25 cases were included in this study. Surgical specimen mistakes were indicated in all grades of the SPSM process, in the intraoperative phase during specimen ordering $(8 \% ; n=2)$, labeling $(24 \% ; n=6)$, containment $(4 \% ; n=1)$, transport or storage $(40 \% ; n=10)$, quality $(12 \% ; n=3)$, processing $(4 \%$; $\mathrm{n}=1)$ and collection $(8 \% ; \mathrm{n}=2)$. The results described cases in more than one grade of the process. It's possible that a case affected more than one contributing parameter (Tables 1 and 2). Twenty five surgical specimen events were defined in all grades of the SPSM process during the intraoperative phase and, particularly, with labeling, collection/ preservation, and transport in specimen. The most common six pieces contributing parameters were mistakes in communication errors, staff carelessness, staff poor awareness, lack of information, and lack of policies/protocols issues, order and documentation problems. It's possible that a case affected more than one contributing parameter (Table 3). In this study; a contributing parameter may affect more than one case such as staff carelessness cause either specimen collection or labeling as well as other all grades, likewise staff poor awareness. However; transport and/or storage of specimen events was determined generally related with lack of policies/protocols. Requirements for containment were met for using appropriate of size the container. Neutral Buffered Formalin (NBF) was used to preservation solution. Identification and labeling are the processes of affixing information that

\begin{tabular}{|c|c|}
\hline Surgical Specimen Classification & Casesa $\mathbf{n = 2 5}(\mathbf{\%})$ \\
\hline Ordering & $2(8)$ \\
\hline Specimen collection & $2(8)$ \\
\hline Specimen containment & $1(4)$ \\
\hline Specimen labeling & $6(24)$ \\
\hline Transport and/or storage of specimen & $10(40)$ \\
\hline Specimen processing & $1(4)$ \\
\hline Specimen quality & $3(12)$ \\
\hline
\end{tabular}

Table 1: Surgical specimen cases by group.

\begin{tabular}{|c|c|c|}
\hline $\begin{array}{l}\text { Grade in Process } \\
\text { (\%) } n=14\end{array}$ & Problem of section & $\begin{array}{c}\text { Casesa } \\
n=25(\%)\end{array}$ \\
\hline Ordering $2(8)$ & $\begin{array}{l}\text { (Manual typing order used. Wrong or not } \\
\text { clarified patient information) }\end{array}$ & $2(8)$ \\
\hline \multirow{2}{*}{$\begin{array}{l}\text { Specimen collection } \\
2(8)\end{array}$} & Unprotected the integrity of the specimen & $1(4)$ \\
\hline & Wrong technique used & $1(4)$ \\
\hline $\begin{array}{c}\text { Specimen } \\
\text { containment } 1(4)\end{array}$ & Inadequate preservative or solution used & $1(4)$ \\
\hline \multirow{4}{*}{$\begin{array}{l}\text { Specimen labeling } \\
\qquad 6(24)\end{array}$} & $\begin{array}{c}\text { Incorrect or missing patient informative } \\
\text { labeling }\end{array}$ & $2(8)$ \\
\hline & $\begin{array}{l}\text { Mislabeled: Label or requisition uncertain, } \\
\text { incomplete, or unclarity (eg, diagnosis, } \\
\text { biochemical hazard information, time/date ) }\end{array}$ & $2(8)$ \\
\hline & Mislabeled: Wrong side and tissue identified & $1(4)$ \\
\hline & Label and requisiton not match & $1(4)$ \\
\hline $\begin{array}{l}\text { Transport and/or } \\
\text { storage of specimen } \\
10(40)\end{array}$ & More than one hour delay, Not cooled & $10(40)$ \\
\hline $\begin{array}{c}\text { Specimen } \\
\text { processing } 1(4)\end{array}$ & $\begin{array}{l}\text { Reversal from the laboratory(eg, breakage, } \\
\text { lost, error in specimen) }\end{array}$ & $1(4)$ \\
\hline \multirow{3}{*}{$\begin{array}{l}\text { Specimen quality } \\
\qquad 3(12)\end{array}$} & Quantity insufficient & $1(4)$ \\
\hline & Contamination risk & $1(4)$ \\
\hline & Degenerate specimen & $1(4)$ \\
\hline
\end{tabular}

Table 2: Kinds of surgical specimen problems by grade of specimen management

there are the name, surname, citizenship id number, date of birth and file number the patient, doctor information, no bio-hazard warning on the electronic label. Devices intended for transport were not labeled to communicate chemical and biohazard information. Specimens were not transported rapidly to the pathology laboratory. After containment, specimens had been kept between 3 hours 46 minutes and 18 minutes at room (Table 4). Temperature for about 20 to 23 centigrade degrees in the operating room where shelving unit. In the operating room, specimens were transported to pathology laboratory twice a day at 10 and 14 o'clock. It was determined that no use of a guideline about specimen management by the hospital, all of the health workers and personnel were realized the specimen management by individual knowledge and experiences.

\section{Discussion}

This research a point prevalence survey examines realization process of SPSM. In this study, all intervention and errors were observed one to one during a day by the researchers during the intraoperative phase. This study provides an important network of knowledge on the types of mistakes about working style in the operating room and strives to represent most common errors and quality improvement priority. Identified errors in this study this that labeling, containment, transfer errors, specimen and occupational safety, record, briefing, verification and control deficiency have risk of cause serious potential problems 


\begin{tabular}{|c|c|}
\hline \multicolumn{2}{|c|}{ Surgical Pathology Specimen Cases by Contributing Parameters } \\
\hline Contributing parameter & Casesa (\%), $\mathbf{n = 2 5}$ \\
\hline Comunication errors & $2(3.92)$ \\
\hline Staff carelessness & $7(13.72)$ \\
\hline Lack of information & $3(5.88)$ \\
\hline Lack of policies/protocols & $16(31.37)$ \\
\hline Order and documentation problems & $3(5.88)$ \\
\hline Staff poor awareness & $20(39.21)$ \\
\hline
\end{tabular}

Table 3: Surgical specimen cases by contributing parameter.

\begin{tabular}{|c|c|}
\hline Surgical Specimens & Retention Time \\
\hline Nevus excision 1st & 2 hours, 53 minutes \\
\hline Nevus excision 2nd & 3 hours, 46 minutes \\
\hline Back mass excision & 1 hour \\
\hline Pilonidal sinus excision & 18 minutes \\
\hline Transurethral resection (TUR-M) 1st & 1 hour, 50 minutes \\
\hline Transurethral resection (TUR-M) 2nd & 1 hour, 3 minutes \\
\hline Transurethral resection (TUR-M) 3rd & 18 minutes \\
\hline Transurethral resection of the prostate (TUR-P) 1st & 2 hours \\
\hline Transurethral resection of the prostate (TUR-p) 2nd & 1 hours \\
\hline Carinal resection-Adenoid cystic carcinoma 1st & 40 minutes \\
\hline Carinal resection-Adenoid cystic carcinoma 1st & 1 hour, 20 minutes \\
\hline Laparoscopic cholecystectomy resection & 3 hours, 18 minutes \\
\hline Gastric sleeve resection (GSR) & 50 minutes \\
\hline Soft tissue tumor resection & 1 hour \\
\hline
\end{tabular}

Table 4: Retention time of the specimens before being transported to the pathology laboratory.

during the intraoperative processes. In this study; it was determined 25 pieces preventable events during the SPSM process of followed 14 surgical pathology specimens which 4 excisions, 10 resections. These events were observed that most common in transport and/or storage of specimen $(40 \%)$ and labeling (24\%) as well as the contributing factor is staff poor awareness(39\%), also; longest held materials on inappropriate conditions more than 3 hours. The results of study provide a rich information source on the kinds of mistakes that take place and the parameters contritubing to those mistakes. These cases can have important influence on quality of patient health care and consequences, comprising the need for extra interventions, treatment, health care, also new procedures, an increased hospitalization, or as well operation. The perioperative nurses should evaluate specimen management requires which include identifying who requires being notified, how the specimen will be collected, handled, how the specimen transferred from the sterile field, how the specimen will be contained and transported, what documentation is needed for safe, effective, appropriate SPSM [1,3-5]. During the preoperative patient assessment, the perioperative registered nurse should identify the site of the specimen to be collected [1]. Using the "Write down, read back" technique should verify the identification of the specimen between the surgeon and circulator nurse [4]. In this study, it is clear that not keeping the desirable collaboration and communication between perioperative nurses and another multidisciplinary team members. Using a guidance, recommended practices for effective SPSM provides to help perioperative nurses in collaboration with a multidisciplinary team. It was observed in this study that not using a guidance about SPSM by the perioperative nurses. Most common, literature reviews showed that surgical specimens were not approved in the laboratory, having been either preventable or temporarily/permanently lost $[7,8,10,11]$. Therefore, technological solutions, such as assorted systems of bar code and radiofrequency familiarization, have been tested to reduce errors during all stages of the SPSM process and can be very beneficial [10]. Some researches described problems in SPSM process and analysis in the laboratory. These results are steady with previous researches reporting mistakes in the SPSM process ranging from $0.01 \%$ to $51 \%$ of the overall total mistakes [5,7-11]. In our study, $60 \%$ (15 of 25) of the errors were smilar issues and intraoperative team members borne. However, in 40\% (10 of 25) of cases observed by managers borne SPSM mistakes may be resulted in the need for extra procedures. Thanks to the results of this study was obtained significant evidence for SPSM process of systems weaknesses within the context of the health care organization. Preservation specimen integrity and antigenicity in pathology specimens is the essential stair in comletely diagnoses and $10 \%$ neutral buffered formalin (NBF) has been a traditional preservative $[12,13]$. Preservation of the specimen helps prevent decomposition, inhibit microbial growth, and prevent autolysis $[13,14]$. In this study specimen safety was proved with ideal solution. The Occupational Safety and Health Administration provide guidelines for storage and handling of NBF. Perioperative nurses play a crucial role to patient advocacy and corporation with other perioperative team members a safe perioperative environment [14]. Specimens container label should be contain patient communication, specimen, protective and biohazard information [1]. When specimen transport will not occur immediately, specimens must be temporarily stored in equipment that will maintain correct temperature to optimally maintain the specimen [1]. In this study, all specimens were held between 18 minutes and 3 hours, 46 minutes in a shelving unit at room temperature for about 20 to 23 centigrade degrees in the operating room (Table 4). It was a risky practice for safe SPSM as well as no an evidence-based application. Safe SPSM is required influential multidisciplinary communication, to prevent disorganization, and awareness of the possible error and previous study were reported most common surgical specimen mistakes take place in the pre-laboratory phase [15]. This study is coherent with other researches [5,7,9-11]. SPSM a multidisciplinary and multifunctional process so the margin of error is high. In the end, unsafe SPSM may lead to the need for additional procedures. It is right for surgical patients that best practices including patient safety to every patient while taking health care. This study of data was collected by the researchers in person. Routine working was getting around to review by the researchers and these results rather new collected in 2016. Therefore, this study of reliability is substantially high but it was approved as a limitation in order to realize a single center.

\section{Conclusion}

Safe SPSM is an essential part of patient and occupational safety. Therefore, it should be primarily determined that giving a break to safe SPSM in corporate policies and procedures. In this context of best practice that every hospital should be used a guidance which met expectations at an optimal level when provided patient safety. It provides to seen errors which are taken notes all practices that include correct and inaccurate. Each of the one important factor for safe SPSM that developed by information and experiences belonging to health care workers. It should be returned one each policy in a manner that provides to be trained and awareness the importance of SPSM to health care and support workers. It should be recorded accurate and complete in SPSM process. It will be a step that increased the number of the new study in literature for defining errors. Health care workers should willing to learn patient safety and developed of corporate culture in the operating room.

\section{References}

1. Graybill-D'Ercole P (2014) RP Implementation: specimen management. AORN 
Citation: Bulbuloglu S, Eti Aslan F, van Giersbergen MY(2018) The Surgical Pathology Specimen Safety Study: Management Errors in the Operating Room. Surgery Curr Res 8: 305. doi: 10.4172/2161-1076.1000305

Page 4 of 4

J 100: 625-633.

2. Shirey C, Perrego K (2015) Standardizing the handling of surgical specimens. AORN J 102: e1- 10.

3. Van Wicklinm SA (2015) Back to basics: specimen management. AORN J 101: 558-563.

4. SteelmanVM, Williams TL, Szekendi MK, Halverson AL, Dintzis SM, et al. (2016) Surgical specimen management: A descriptive study of 648 adverse events and near misses. Arch Pathol Lab Med 140: 1390-1396.

5. Seferian EG, Jamal S, Clark K, Cirricione M, Burnes-Bolton L, et al. (2014) A multidisciplinary, multifaceted improvement initiative to eliminate mislabelled laboratory specimens at a large tertiary care hospital. BMJ Qual Saf 23: 690697

6. Li JK, Shah BA (2014) Survey on imaging management and handling of breast surgical specimens by radiologists. J Am Coll Radiol 11: 890-893.

7. Dirimeşe E, Ozşaker E (2015) Tissue samples-related practices. Yavuz Van Giersbergen M, Kaymakçı, S. Intraoperating Nurse, Turkish Surgical and Operating Room Nurses Published. Izmir, pp: 567-569

8. Bulbuloglu S, Sevin K, Çakır S, Aslan FE (2017) Safe surgical pathology material management in the operating room. Health and Nursing Management J $4: 37-42$.

9. Bulbuloglu S, Kapıkıran G, Aslan FE (2017) Erros in the surgical pathology specimen managament: A meta analisys. 2nd International, 10th nationa congress of Turkish surgical and operating room nurses Antalia, Turkey.

10. Snyder SR, Favoretto AM, Derzon JH, Christenson RH, Kahn SE, et al. (2012) Effectiveness of barcoding for reducing patient specimen and laboratory testing identification errors: A laboratory medicine best practices systematic review and meta-analysis. Clin Biochem 45: 988-998.

11. Martis WR, Hannam JA, Lee T, Merry AF, Mitchell SJ (2016) Improved compliance with the World Health Organization surgical safety checklist is associated with reduced surgical specimen labelling errors. N Z Med J 129 63-67.

12. Simionescu O, Blum A, Grigore M, Costache M, Avram A, et al. (2016) Learning from mistakes: Errors in approaches to melanoma and the urgent need for updated national guidelines. Int J Dermatol 55: 970-976.

13. Bell WC, Young ES, Billings PE, Grizzle WE (2008) The efficient operation of the surgical pathology gross room. Biotech histochem 83: 71-82.

14. Costa S, Coelho P, Costa C, Silva S, Mayan O, et al. (2008) Genotoxic damage in pathology anatomy laboratory workers exposed to formaldehyde. Toxicology 252: $40-48$.

15. Goldsmith JD, Siegal GP, Suster S, Wheeler TM, Brown RW (2008) Reporting guidelines for clinical laboratory reports in surgical pathology. Arch Pathol Lab Med 132: 1608-1616 